\title{
Cloning, characterization and phylogenetic analyses of members of three major venom families from a single specimen of Walterinnesia aegyptia, 岤, 动动
}

\author{
Hsin-Yu Tsai ${ }^{a}$, Ying Ming Wang ${ }^{a}$, Inn-Ho Tsai ${ }^{a, b, *}$ \\ a Institute of Biological Chemistry, Academia Sinica, P.O. Box 23-106, Taipei, Taiwan \\ ${ }^{\mathrm{b}}$ Graduate Institute of Biochemical Sciences, National Taiwan University, Taiwan
}

\section{A R T I C L E I N F O}

\section{Article history:}

Received 18 December 2007

Received in revised form

14 February 2008

Accepted 20 February 2008

Available online 29 February 2008

Keywords:

Phospholipase $\mathrm{A}_{2}$

Three-finger toxin

Kunitz protease inhibitor

cDNA cloning and sequencing

Walterinnesia venom

\begin{abstract}
A B S T R A C T
Walterinnesia aegyptia is a monotypic elapid snake inhabiting in Africa and Mideast. Although its envenoming is known to cause rapid deaths and paralysis, structural data of its venom proteins are rather limited. Using gel filtration and reverse-phase HPLC, phospholipases $\mathrm{A}_{2}$ (PLAs), three-fingered toxins (3FTxs), and Kunitz-type protease inhibitors (KIns) were purified from the venom of a single specimen of this species caught in northern Egypt. In addition, specific primers were designed and PCR was carried out to amplify the cDNAs encoding members of the three venom families, respectively, using total cDNA prepared from its venom glands. Complete amino acid sequences of two acidic PLAs, three short chain 3FTxs, and four KIns of this venom species were thus deduced after their cDNAs were cloned and sequenced. They are all novel sequences and match the mass data of purified proteins. For members of each toxin family, protein sequences were aligned and subjected to molecular phylogenetic analyses. The results indicated that the PLAs and a Kunitz inhibitor of $W$. aegyptia are most similar to those of king cobra venom, and its 3FTxs belongs to either Type I $\alpha$-neurotoxins or weak toxins of orphan-II subtype. It is remarkable that both king cobra and $W$. aegyptia cause rapid deaths of the victims, and a close evolutionary relationship between them is speculated. (c) 2008 Elsevier Ltd. All rights reserved.
\end{abstract}

\footnotetext{
Abbreviations: PLA, phospholipase $\mathrm{A}_{2}$; $3 \mathrm{FTx}$, three-finger toxin; KIn, Kunitz-type protease inhibitor; dPPC, dipalmitoyl glycerophosphocholine; HPLC, high performance liquid chromatography; Wa, Walterinnesia aegyptia.

The sequence data were deposited in the GenBank database with the following accession numbers: EU196553-EU196554 for Wa-PLII and Wa-PLI, EU196555-EU196557 for Wa-III, Wa-IV and Wa-V, EU196558EU196561 for KIn-I, III, II, and IV.

Ethical statement: The experiments carried out in the present report did not involve live animals.

* Corresponding author at: Institute of Biological Chemistry, Academia Sinica, P.O. Box 23-106, Taipei, Taiwan. Tel.: +8862 23620264 ; fax: +886223635038

E-mail address: bc201@gate.sinica.edu.tw (I.-H. Tsai).
}

\section{Introduction}

Walterinnesia aegyptia (black desert cobra) is a monotypic species distributed over the desert areas of Egypt, Israel, Syria, Iraq, Iran, Saudi Arabia, and Turkey. Like other elapid venoms, its envenoming causes muscular paralysis and respiratory failure in mice and human victims (Ismail et al., 1998; Lee et al., 1976; Lee and Tsai, 1972; Samejima et al., 1997). Notably, injection of lethal doses of its venom on rats or mice caused rapid deaths. Previously, two neurotoxic three-finger toxins (3FTxs) of $W$. aegyptia venom from Israel have been purified and sequenced, but cobra cardiotoxin-like proteins were not detected (Lee et al., 1976; Samejima et al., 1997). Being the nonenzymatic markers of elapid venom, the 3FTxs form a superfamily that has a stable three- $\beta$ sheets scaffold. They 
display a broad array of known or undefined functions, e.g. antagonism of various subtypes of nicotinic acetylcholine receptors, cytotoxicity or cardiotoxicity, inhibition of Ltype calcium channel, and acetylocholinesterase (Kini, 2002; Tsetlin and Hucho, 2004).

Another common non-enzymatic component of elapid venom is Kunitz protease inhibitor (KIn), which has never been studied in the $W$. aegyptia venom. In addition, the venom of $W$. aegyptia was found to contain various enzymes including phospholipases $\mathrm{A}_{2}$ (PLAs) (Simon and Bdolah, 1980), L-amino acid oxidase, and proteolytic enzymes (Gitter and de Vries, 1968). In fact, two acidic PLAs of $W$. aegyptia venom have been purified but their sequences were not reported (Simon and Bdolah, 1980). The 13-14 kDa PLAs hydrolyze phospholipids and release fatty acids and lysophospholipids in $\mathrm{a} \mathrm{Ca}^{2+}$-dependent manner (Danse et al., 1997).

The aims of this study are to investigate the proteome and the transcriptome of venom components of $W$. aegyptia and to understand the biosystematics of this elapid species. Here, three low molecular weight and common venom families (i.e. PLA, 3FTx, and KIn) would be focused. We have purified and characterized the venom proteins of a single specimen of $W$. aegyptia from Egypt. In the meantime, we used PCR and facile cDNA cloning to study the transcriptome of each of the three venom families. The cDNA-deduced sequence data were matched to those of the purified proteins. These sequences were then subjected to phylogenetic analyses to expand our knowledge about the elapid systematics. Structural features of the $W$. aegyptia proteins would also be discussed for a better understanding of the structure-function relationships.

\section{Materials and methods}

\subsection{Materials}

Crude venom was milked from a single specimen of $W$. aegyptia (northeastern Egypt). Two days later its venom glands were dissected after sacrificing the snake. The tissue was preserved for several weeks in the RNAlater solution (Ambion, USA) before total RNA was extracted. Modification and restriction enzymes and the pGEM-T vectors were purchased from Promega Corp. (Madison, USA). Synthetic dipalmitoyl phosphatidylcholine was from Avanti-Biochemical (Alabama, USA). Triton $\mathrm{X}-100$ and sodium deoxycholate were from Sigma Chemical Co. (USA). Other chemicals used were of reagent grade.

\subsection{Purification and characterization of venom proteins}

Lyophilized venom was dissolved in a small volume of $100 \mathrm{mM} \mathrm{CH}_{3} \mathrm{COONH}_{4}$ (pH 6.24), followed by centrifugation. The supernatant was applied to a Superdex-G75 gel filtration column and eluted with the same buffer on a FPLC system. Fractions containing PLAs were pooled and lyophilized before further purification by reverse-phase HPLC. After being injected into a Vydac column of C18 silica gel $(4.6 \times 250 \mathrm{~mm})$, the sample was eluted by a gradient of buffer A and B, made of $0.07 \%(\mathrm{v} / \mathrm{v})$ trifluoroacetic acid and acetonitrile, respectively. Proteins collected were dried in a vacuum-centrifuge device (Labconco, USA). We also assumed that the averaged extinction coefficients at $280 \mathrm{~nm}$ for fractions A and B were approximately twice those for fractions $C$ and $D$ of the gel filtration profile. The relative content $(\%, w / w)$ of each protein in the crude venom was estimated, based on the relative absorbance at $280 \mathrm{~nm}$ of the area under the four fractions collected in gel filtration (Fig. 1) as well as the relative absorbance at $230 \mathrm{~nm}$ of each HPLC peaks (Fig. 2). The molecular weight of each purified protein was analyzed by QSTAR XL Nano-ESI Mass Spectrometer System (Applied Biosystems) in the protein-core facility of our institute.

\subsection{Assays of PLAs}

Micelles of $3 \mathrm{mM}$ L-dipalmitoyl phosphatidylcholin ( $\mathrm{diC}_{16} \mathrm{PC}$ ) with $3 \mathrm{mM}$ sodium deoxycholate or $6 \mathrm{mM}$ Triton $\mathrm{X}-100$, and $100 \mathrm{mM} \mathrm{NaCl}$ were prepared in a glass-Teflon tissue homogenizer, and $2.5 \mathrm{ml}$ of the solution was transferred to the thermostated reaction cup of a $\mathrm{pH}$-stat apparatus (Radiometer, Copenhagen, Denmark). With constant stirring, a small aliquot of $\mathrm{CaCl}_{2}$ was added to a final concentration of $10 \mathrm{mM}$ just before the addition of enzyme. With $\mathrm{pH}$ set to 7.40 , the release of acid during substrate hydrolysis was followed by titration with $8 \mathrm{mM}$ $\mathrm{NaOH}$ at $37^{\circ} \mathrm{C}$. The initial hydrolysis rate was corrected for the non-enzymatic rate in each experiment.

\subsection{Cloning and sequencing of venom toxins}

The RNA from $W$. aegyptia venom glands was extracted using mRNA extraction kit. Their complementary DNAs

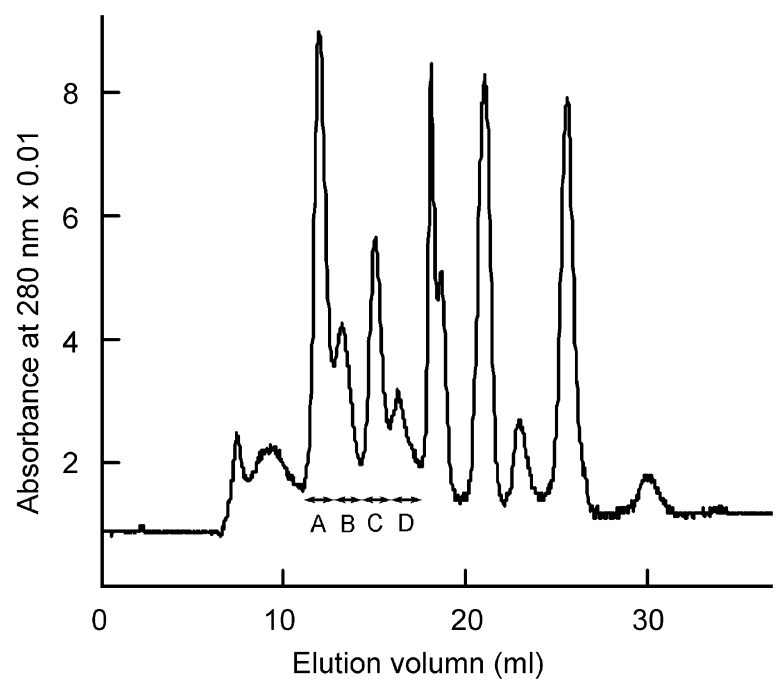

Fig. 1. Separation of $W$. aegyptia venom proteins by gel filtration. The venom powder $(1.5 \mathrm{mg}$ ) was dissolved in $100 \mu \mathrm{l}$ water and loaded onto a Superdex G75 (HR10/30) column pre-equilibrated with $0.1 \mathrm{~N}$ ammonium acetate buffer ( $\mathrm{pH} 7.1)$. Elution of venom proteins was carried out on a FPLC system at a flow rate of $1 \mathrm{ml} / \mathrm{min}$ and monitored at $280 \mathrm{~nm}$. Fractions were collected separately and lyophilized. 


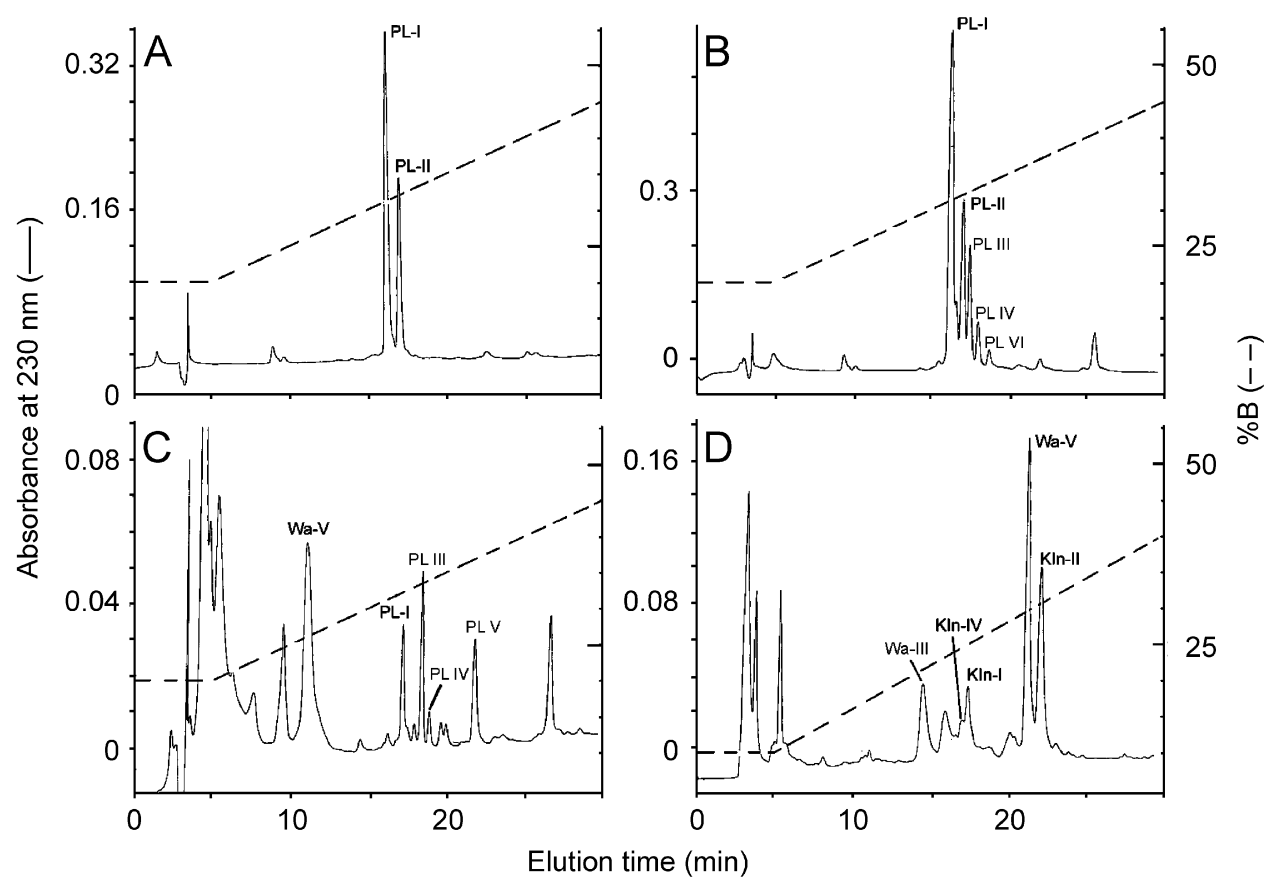

Fig. 2. Purification of venom proteins by RP-HPLC. Proteins obtained from fractions A-D in the gel filtration (Fig. 1) were re-dissolved and injected into a Vydac RP-C18 column separately. The elution procedure started with $20 \%$ buffer B for 5 min followed by a gradual increase of buffer B for 25 min, at a flow rate of $1 \mathrm{ml} / \mathrm{min}$ to establish a concentration gradient (dashed lines). Eluted polypeptides were monitored at $230 \mathrm{~nm}$. Annotations of the proteins are as shown in Table 1.

(cDNAs) were prepared using cDNA synthesis kit according to the manufacturer's instructions (Stratagene, USA). It is known that snake venoms undergo positive Darwinian evolution (Ohno et al., 1998), which features high conservation at the $5^{\prime}$ and $3^{\prime}$ untranslated regions (UTR) but rapid evolution of the exon regions. Using primers designed from conserved UTR sequences, we were able to amplify specific cDNAs by PCR, and clone various venomspecific cDNAs. PCR primers were synthesized based on the conserved UTR and/or signal peptide regions of the cDNA sequences previously published for elapid venom PLAs (Tsai et al., 2002), 3FTxs (Chang et al., 1999) and KIns (Chang et al., 2001). To amplify the 3FTx cDNAs, Primer 1 used was 5'-ATGAAAACTCTGCTGCTGWCCTTG-3' and Primer 2 was 5'-CTCAAGGAAWTTAGSCACTCRKAGAG-3'. To amplify the PLA CDNAs, Primers 3 and 4 used were 5'GCAGTTTGTGTCTCC CTCTTAGGA-3' and 5'-CACAGTCCTTGAGCTGAAGCTTCTC-3', respectively. To amplify the KIn cDNAs, Primers 5 and 6 used were 5'-CCAGACGGCTTCATCATG-3' and 5'-AAAAGGAATRATCCAGG-3', respectively. Primers 1,3 , and 5 were in the sense orientation of the $5^{\prime}-$ end sequences, whereas primer 2,4 , and 6 were in the antisense orientation of the 3'-end UTR.

PCR (Mullis and Faloona, 1987) was conducted using the cDNAs derived from the venom glands as templates and SuperTaq DNA polymerase (HT Biotech, UK) was used. Conditions of each of the 35 cycles were set to $92{ }^{\circ} \mathrm{C}$ for $1.0 \mathrm{~min}$ (denaturation), $52^{\circ} \mathrm{C}$ for $1.0 \mathrm{~min}$ (annealing), and $72{ }^{\circ} \mathrm{C}$ for $1.0 \mathrm{~min}$ (extension). After amplification, the DNA fragments of expected size were resolved by $1 \%$ agarose gel electrophoresis and harvested. After treating with polynucleotide kinase, they were inserted into the pGEM-
T vector (Promega Biotech), and then used to transform Escherichia coli strain JM109 (Maniatis et al., 1989). The plasmid DNA was extracted from white transformants and its restriction pattern was examined by agarose gel electrophoresis. Each cloned cDNA was sequenced by the DNA-Sequencing-System (model 373A, PE-Applied Biosystems, USA). The sequences were confirmed at least twice and translated into amino acid sequences.

\subsection{Phylogenetic trees}

The amino acid sequences were aligned using the CLASTAL W (Thompson et al., 1994). Cladograms were constructed based on the aligned sequences by a neighbor-joining algorithm using the PHYLIP program (Felsenstein, 1992), degree of confidence for the internal lineage was determined by bootstrap methods (Felsenstein, 1985).

\section{Results and discussion}

\subsection{Venom protein purification and characterization}

The $W$. aegyptia venom collected from an individual snake was in low quantity. It was first separated by gel filtration. As shown in Fig. 1, four fractions (A-D) contained most of the venom proteins were harvested before $20 \mathrm{ml}$ of the elution volume and lyophilized. The later peaks were also analyzed but gave poor yields of proteins. By HPLC, PLAs were further purified from fractions $A$ and $B$, while the 3FTxs and KIns (6-7 kDa) were purified from fractions $C$ and D (Fig. 2). Molecular 
Table 1

Characterization and enzymatic activities of purified venom PLAs of $W$. aegyptia

\begin{tabular}{|c|c|c|c|c|}
\hline \multirow[t]{2}{*}{ PLA } & \multirow{2}{*}{$\begin{array}{l}\text { Content } \\
\%(w / w)\end{array}$} & \multirow{2}{*}{$\begin{array}{l}\text { Mass } \\
\text { (Da) }\end{array}$} & \multicolumn{2}{|c|}{ Specific activity ( $\mu \mathrm{mol} / \mathrm{mg} / \mathrm{min})$} \\
\hline & & & $\begin{array}{l}\text { DPPC plus } \\
\text { deoxycholate }\end{array}$ & $\begin{array}{l}\text { DPPC plus } \\
\text { Triton X-100 }\end{array}$ \\
\hline PL-I & 13.0 & 13,342 & $793 \pm 27$ & $591 \pm 3.3$ \\
\hline PL-II & 6.0 & 13,343 & $54.1 \pm 3.6$ & $15.0 \pm 0.1$ \\
\hline PL-III & 1.0 & 13,455 & n.d. & n.d. \\
\hline PL-IV & 1.5 & 13,562 & n.d. & n.d. \\
\hline PL-V & 0.2 & 14,206 & n.d. & n.d. \\
\hline
\end{tabular}

Table 2

Characterization of purified 3FTx and KIn of W. aegyptia venom

\begin{tabular}{llll}
\hline Protein & $\begin{array}{l}\text { Content } \\
\%(\mathrm{w} / \mathrm{w})\end{array}$ & $\begin{array}{l}\text { Mass } \\
(\mathrm{Da})\end{array}$ & N-terminal sequence 1-16 \\
\hline $\begin{array}{l}\text { (3FTx) } \\
\text { Wa-III }\end{array}$ & 2.0 & 6852 & FVCHNQQSSQPPTTTN \\
Wa-V & 2.4 & 7400 & LTCLICPKKYCNQVHT \\
(KIn) & & & \\
KIn-I & 0.2 & 6347 & RPGLCELPAETGPCKA \\
KIn-II & 0.8 & 6394 & RPRLCELPAESGLCNA \\
KIn-IV & 0.4 & 6779 & LGGPKYCHLPADPGPC \\
\hline
\end{tabular}

mass of each purified protein was determined by ESI-Mass spectrometry (Tables 1 and 2). The esterase activities of Wa PL-I and PL-II were also assayed by pH stat method (Table 1). There are another three PLA-like components, which could be purified at very low yields. Some of the purified proteins were subjected to automatic sequencing by protein sequencer (Tables 1 and 2).

We also used azocasein to assay the protease activities in $W$. aegyptia venom. The activities were greatly reduced to control level when $5 \mathrm{mM}$ EDTA was added to inhibit the proteases (data not shown). Thus, W. aegyptia venom probably contains metalloprotease.

\subsection{Cloning and sequencing}

The primers used in our PCR to amplify cDNA of the three families worked well and many of the venom cDNAs were successfully cloned. After sequencing 50 isolated cDNA clones for each of the venom family, distinct sequences for two PLAs, three 3FTxs, and four KIns were obtained. Only those clones that have been confirmed at least twice were included in the results (Table 2). Notably, the 27-residue conserved signal peptides of the PLAs are highly similar to those of the other venom group I PLAs, the 21-residue conserved signal peptides of the 3FTxs are also identical to those of the 3FTxs from other venom species (Chang, 2007). The 24-residue signal peptides of the four Wa-KIns are identical except that of KIn-IV, which contains a V21I substitution. These signal peptides are also very similar to those of the other venom KIns (Zupunski et al., 2003).

The $\mathrm{N}$-terminal sequences and the molecular weights of most of the venom proteins (Tables 1 and 2) could
Table 3

Data for members of three venom families of $W$. aegyptia deduced from their cDNA sequences

\begin{tabular}{|c|c|c|c|}
\hline $\begin{array}{l}\text { Encoded } \\
\text { protein }\end{array}$ & $\begin{array}{l}\text { Calculated } \\
\text { mass }\end{array}$ & $\begin{array}{l}\text { Predicted } \\
\text { pI }\end{array}$ & Signal peptide sequence \\
\hline (PLA) & & & $1-27$ \\
\hline PL-I & 13,342 & 4.6 & $\begin{array}{l}\text { MYPAHLLVLL AVCVSLLGAA } \\
\text { NIPPQPL }\end{array}$ \\
\hline PL-II & 13,343 & 4.6 & $\begin{array}{l}\text { MYPAHLLVLL AVCVSLLGAA } \\
\text { NIPPQPL }\end{array}$ \\
\hline (3FTx) & & & $1-21$ \\
\hline Wa-III & 6853 & $>8.9$ & MKTLLLTLVV VTIVCLDLGYT \\
\hline Wa-IV & 6782 & $>8.7$ & MKTLLLTLVV VTIVCLDLGYT \\
\hline Wa-V & 7400 & $>8.8$ & MKTLLLTLVV VTIVCLDLGYT \\
\hline (KIn) & & & $1-24$ \\
\hline KIn-I & 6347 & 8.6 & $\begin{array}{l}\text { MSSGGLLLLL GLLTLWAELT } \\
\text { PVSG }\end{array}$ \\
\hline KIn-II & 6394 & 8.3 & $\begin{array}{l}\text { MSSGCLLLLL GLLTLWAELT } \\
\text { PVSG }\end{array}$ \\
\hline KIn-III & 6378 & 8.6 & $\begin{array}{l}\text { MSSGCLLLLL GLLTLWAELT } \\
\text { PVSG }\end{array}$ \\
\hline KIn-IV & 6780 & $>9.2$ & MSSGCLLLLL GLLTLWAELT PISG \\
\hline
\end{tabular}

Isoelectric points ( $\mathrm{pI}$ ) and masses were calculated from the predicted protein sequences.

match those predicted from their cDNA sequences (Table 3). However, two cDNA clones appeared to have low expression level and their encoded proteins (Wa-IV and KIn-III) could not be purified. In addition, several minor PLAs were purified and identified (Table 1) from fractions B and C (Fig. 2), but none of the cDNAs we cloned could match their masses determined by ESI-MS.

\subsection{Venom PLA isoforms}

Prior to our study, two non-lethal acidic PLAs from $W$. aegyptia venom have been reported (Simon and Bdolah, 1980). Here we have purified and cloned two acidic PLA variants and designated them as Wa PL-I and PL-II. Similar to the other group IA venom PLAs, both PLA precursors have 14-conserved Cys residues in the mature protein of 120 residues. A BLASTP search (Altschul et al., 1997) identified a PLA from king cobra venom (Xu et al., 2003) as the most similar enzyme to Wa PL-I and PL-II. Complete amino acid sequences of Wa PL-I and PL-II and other homologous PLAs were aligned and compared in Fig. 3.

Both Wa PL-I and PL-II contain active site residues H48, D49, Y52, and D99, $\mathrm{Ca}^{2+}$-binding loop at positions 25-33, and a substrate binding hydrophobic channel comprised of L2, F5, I9, W19, Y66, A102, and F106 (White et al., 1990). However, the N-terminal residues H1, G3, N6 are not frequently present in the elapid venom PLAs, although $\mathrm{H} 1$ and N6 have been found in myotoxic and neurotoxic PLAs of pitvipers' venom (Tsai et al., 2004; Chen et al., 2004), and $\mathrm{N} 6$ is also present in bovine pancreatic PLA. Previous mutagenesis study on pancreatic PLA showed that W3A point mutation resulted in decreased catalysis (Liu et al., 1995). Thus small amino acids at position 3, such as G3, might not be favorable for the enzymatic activity. Wa PL-I and II differ by only four substitutions (T70R, S101L, 


\begin{tabular}{|c|c|}
\hline & $\begin{array}{r}50 \\
\times+1 \\
\end{array}$ \\
\hline PL-I & LGQF \\
\hline--11 & $\cdots$ \\
\hline 3. 0 & रLTE. \\
\hline$N$ & . V. - . RS . . A A. \\
\hline H. $h$ & .V. - . RS . . H.AN \\
\hline I & 2. .V. -NRS. .H.A. \\
\hline & $. H . V .-. R$. \\
\hline KBf-VI & AGT--RT . LAYVK. \\
\hline
\end{tabular}

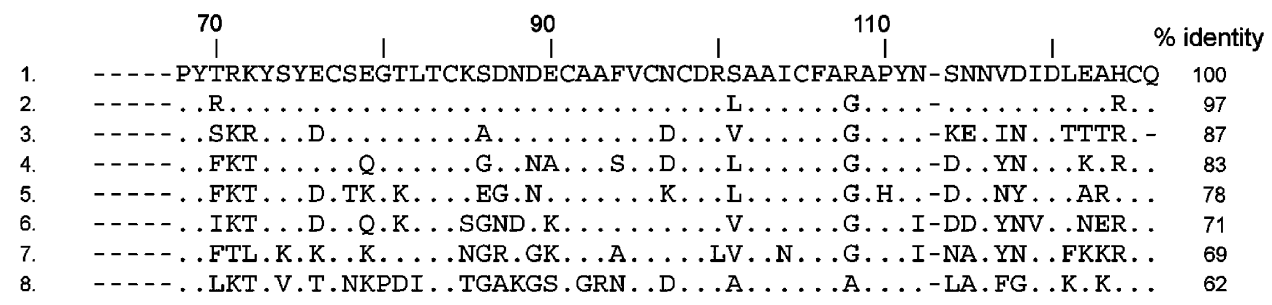

Fig. 3. Alignment of amino acid sequences of Wa-PLAs and related venom PLAs. Their GenBank or SwissProt accession numbers are shown in legend of Fig. 5. Single-letter codes of amino acids are used, gaps are marked with hyphens, the active sites are marked with asterisks. A commonly used numbering system is adopted (Tsai et al., 2002).

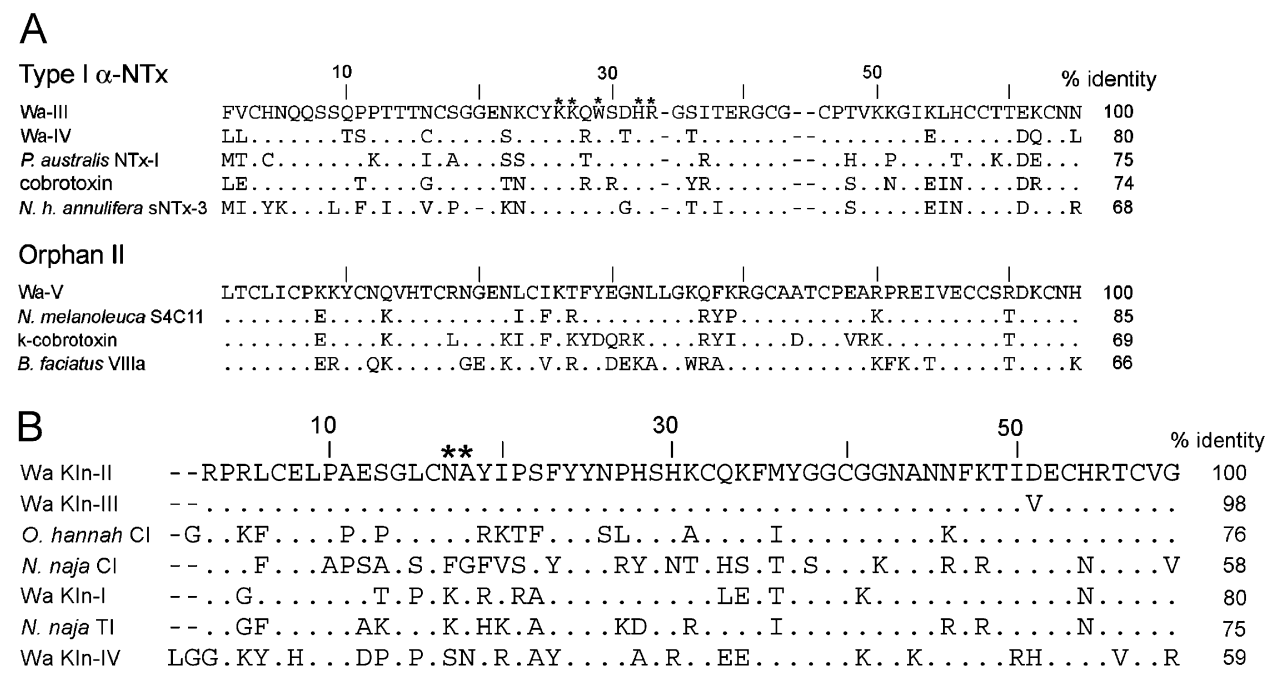

Fig. 4. Multiple alignment of amino acid sequences of 3FTxs and Kunitz inhibitors. (A) Wa-III, Wa-IV, Wa-V and related 3FTxs. (B) Wa KIns and related elapid venom KIns. Their GenBank or SwissProt accession numbers are shown in legends of Figs. 6 and 7, respectively. Single-letter codes of amino acids are used and gaps are marked with hyphens. Important sites were marked with asterisks.

R108G, and H124R), and their predicted pI values of 4.6 are almost identical. However Wa PL-I showed 15-folds higher hydrolytic activity than Wa PL-II in the in vitro assay (Table 1 ), and its content was much higher than that of Wa PL-II (Fig. 2). The reduced enzymatic activity of Wa PL-II could possibly be attributed to the T70R substitution because the 70th residue of other active elapid PLAs are usually neutral, while substitutions L101, G108, and R124 are rather common in other PLAs (Danse et al.,1997).

\subsection{Three-fingered toxins}

We now cloned three novel 3FTxs from the venom of W. aegyptia, and designated the proteins as Wa-III, Wa-IV, and Wa-V (Table 3 ) since the sequences of Wa-III and Wa-
IV were very similar to W-III and W-IV, respectively (Samejima et al., 1997). The sequences of Wa-III, Wa-IV, and Wa-V were aligned in Fig. 4A. Wa-III and Wa-IV are highly basic polypeptides of 62 amino acids, probably containing four conserved disulfide bonds, while Wa-V contains 65 residues with a fifth disulfide bridge in loop I (Tsetlin and Hucho, 2004). The deduced Wa-III sequence differs from the previously reported W-III sequence by two substitutions, namely, S36 and T38 instead of K36 and I38; and the predicted Wa-IV sequence differs from the W-IV sequence by a P30S substitution (Samejima et al., 1997). A special Cys residue is present at position 16 of Wa-IV, which seems to have no significant effect on its toxicity.

Among the 3FTxs sequenced, Wa-III and Wa-IV are about $80 \%$ similar, and they both have conserved residues 
K27, K28, W29, H32, and R33 in loop II (Fig. 4A). It has been known that a W29 and several positively charged residues (e.g. R33) on loop II are important for the postsynaptic neurotoxicities of these $\alpha$-neurotoxins (Pillet et al., 1993; Tremeau et al., 1995; Antil et al., 1999; AntilDelbeke et al., 2000). However, sequence of Wa-V is only $35 \%$ similar to that of Wa-III or Wa-IV, but $85 \%$ identical to that of a cobra weak toxin, Nm S4C11 (Carlsson, 1975).

Previously, three postsynaptically acting neurotoxins (W-III, W-IV, and W-V) were purified from the pooled venom of Isreal $W$. aegyptia and their quantities were in the order of $\mathrm{W}-\mathrm{IV}>\mathrm{W}-\mathrm{V}>\mathrm{W}$-III (Lee et al., 1976). For mice, the medium lethal doses $\left(\mathrm{LD}_{50}\right)$ of $\mathrm{W}$-III and W-IV previously determined were 0.95 and $0.14 \mu \mathrm{g} / \mathrm{g}$, respectively (Samejima et al., 1997). Here, both Wa-III and Wa-V were purified from a single $W$. aegyptia venom (Fig. 2) and the content of $\mathrm{Wa}-\mathrm{V}$ is about 5-fold higher than that of Wa-III (Table 2), but Wa-IV was hardly detectable (Fig. 2C and D). Apparently, differential expression of the 3FTxs variants may cause individual or geographic variations in the toxin proportions and affect the snakebite symptoms.

\subsection{Kunitz-type inhibitors}

We have cloned and sequenced four Kunitz inhibitors from $W$. aegyptia venom, and they are designated as KInI-IV. Except for KIn-III, all of them could be purified from the venom. KIn-I, KIn-II, and KIn-III all contain 57 amino acid residues while KIn-IV contains 59 residues with two extra residues at the $\mathrm{N}$-terminus, similar to some other venom KIns (Fig. 4B). It was shown by in vitro mutagenesis study that removal of the $\mathrm{N}$-terminal three residues do not affect the affinity of a cobra venom KIn for the protease (Cheng et al., 2005). The KIn-II and KIn-III sequences are almost identical except that the D51 in KIn-II is replaced by V51 in KIn-III, however, their sequences are $\leqslant 80 \%$ identical to those of KIn-I and KIn-IV.

Specificities of KIn towards the target protease is usually determined by the main protease contact site (residue 17 in Fig. 4B, short-handed as P1) (Schechter et al., 1967) and its adjacent region (Millers et al., 2006). The P1 site of KIn-I is K17, that of KIn-II and KIn-III is N17 and that of KIn-IV is S17. KIns with K17 or R17 have been classified as trypsin inhibitors, and those with a hydrophobic or aromatic P1-site have been classified as chymotrypsin inhibitors (Laskowski and Kato, 1980). Presumably, Wa KIn-I might inhibit trypsin, while KIn-II and III might inhibit chymotrypsin if both pancreatic enzymes were used in the in vitro assay. However, their true protease targets in vivo remain to be identified.

In addition, the P1' sites of Wa KIn-I-III are all A18. A18 at $\mathrm{P} 1^{\prime}$ position of the inhibitors are highly conserved and is important for their binding affinity to the target protease (Zhou et al., 2004), as shown by the mutational studies of bovine pancreatic trypsin inhibitors (BPTI) (Grzesiak et al., 2000). In contrast, dendrotoxins and other ion-channel binding KIn-like toxins have a charged amino acid at position 18 (Apostoluk and Otlewski, 1998). Remarkably, S17N18 (P1 and P1' sites) is present in KIn-IV, suggesting its possible role as another type of toxin rather than a protease inhibitor. Although KIns from snake venom have been shown to play a pro-coagulation role (Filippovich et al., 2002; Flight et al., 2005), functions and targets of these KIns are interesting subjects to be further investigated.

\subsection{Molecular phylogeny of the three venom families}

Venom proteins are known to undergo accelerated evolutions. Gene duplication followed by variation is an efficient route for venom to evolve into new toxins (Ohno et al., 1998). The efficiency of duplication/variation is suggested by the emergence of several large venom families whose structural scaffolds and/or active sites are conserved or related. The more closely the snakes are related, the more similar the sequences of their genes and proteins. If a sufficient number of related sequences are available, the origin of the venom gene can be determined, or at least their evolutionary history can be inferred.

While viperid and crotalid venoms express only group II PLAs, group I PLAs have evolved in elapid and hydrophiid venoms (Davidson and Dennis, 1990). To find out the evolutionary relationship between $W$. aegyptia and other species, a phylogenetic tree was built based on the amino sequences of the representative group I venom PLAs, using a group-IIB Lys49-PLA (AAR14171) as the outgroup (Fig. 5). The tree topology unveiled the presence of five or six major clusters, including African and Asian cobras, king cobra, kraits, and two or three groups of hydrophiid/Australian elapids. The topology is similar to that of the species tree which was based on mtDNA sequences (Slowinski et al., 1997). Remarkably, the Wa-PLAs are closely associated with a PLA of Ophiophagus hannah venom (Xu et al., 2003) with a high bootstrap score (Fig. 5).

Elapid venom 3FTxs are known to exhibit a great diversity of biological properties (Fry et al., 2003; Karlsson, 1979; Menez, 1998). A phylogenetic tree was also constructed based on 40 amino acid sequences of the short chain 3FTxs (59-64 residues), including the $W$. aegyptia 3FTxs but excluding those of cardiotoxins, while a long chain neurotoxin, $\alpha$-bungarotoxin, was assigned as an outgroup (Fig. 6). This tree reveals that Wa-III and Wa-IV form a unique cluster and are loosely related to the Type I $\alpha$-neurotoxins of other species, which have been known to antagonize muscle nicotinic acetylcholine receptors. Another abundant 3FTx, Wa-V with five disulfide bridges, is closely related to NmS4C11 of Naja mossanbica (Carlsson, 1975) as well as Bf-VIIIa and Bc-wtx from kraits (Fig. 6). They appeared to be weak or poorly characterized toxins, belonging to the orphan subtype-II of a previous 3FTx classification (Fry et al., 2003).

The data set for this tree also includes several newly published sequences of the short chain 3FTxs of Chinese king cobra venom (Li et al., 2006; Rajagopalan et al., 2007). As shown in Fig. 6, king cobra venom expresses at least three kinds of weak 3FTxs, namely orphan type XVIII, orphan type $\mathrm{X}$, and muscarinic toxin. The latter two subtypes are close to those found in venom of African Hemachatus hemachatus (Rinkhals), Aspidelaps scutatus (shield-nose cobra), and Dendroaspis angusticeps (black 


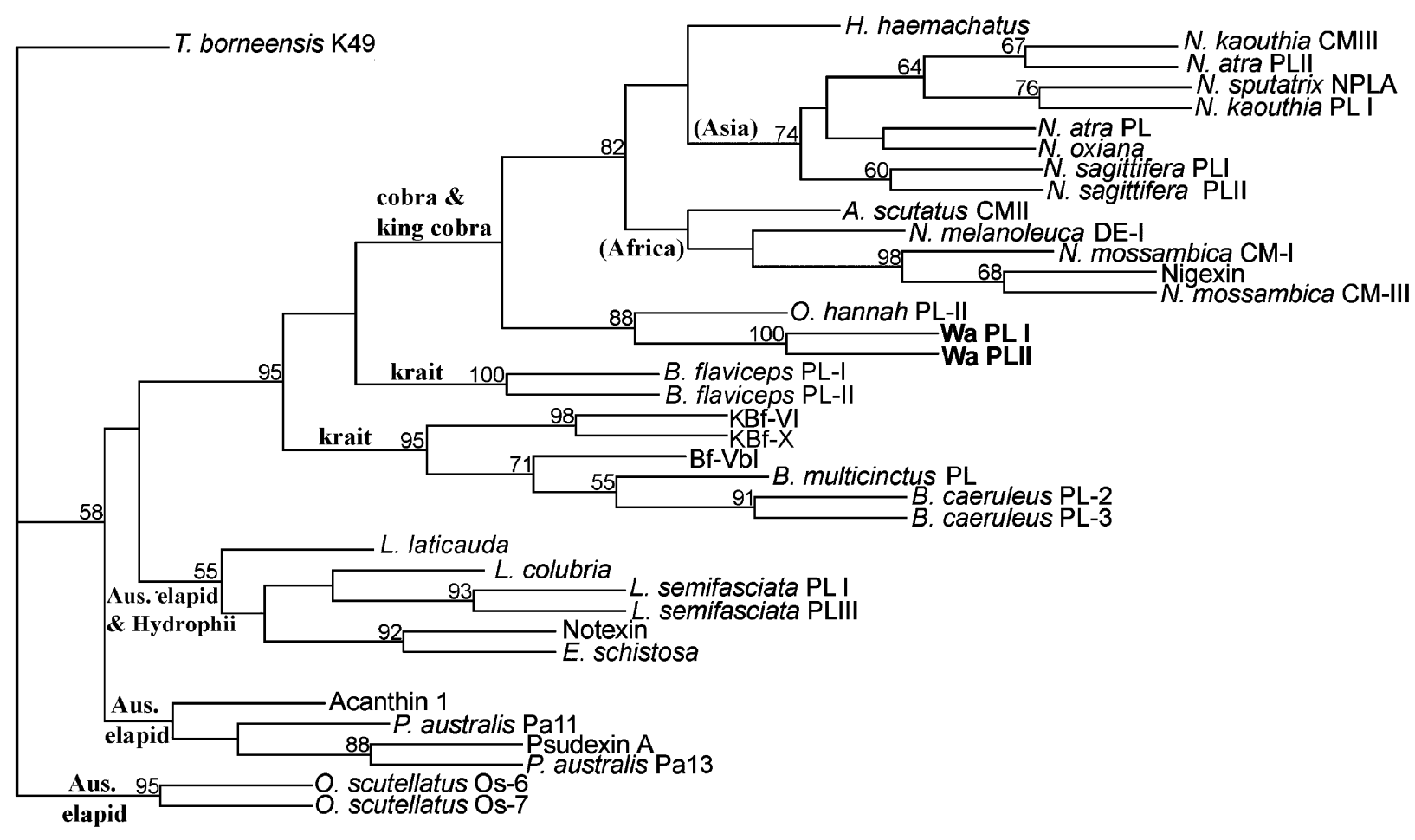

Fig. 5. Phylogenetic analysis of elapid venom PLAs. Trimeresurus borneensis venom Lys49-PLA (AAR14171) is assigned as an outgroup. Bootstrap values, if $>$ 50, are shown at each node. Species, abbreviations, and accession numbers are: Acanthophis antarcticus Acanthin I, P81236; Aspidelaps scutatus CMII, P07037; Bungarus caeruleus PL-2 and 3 AAR19228-AAR19229; Bungarus flaviceps PL-I and II, Ab112359-Ab112360; Bungarus faciatus KBf-VI DQ508411, KBf -X DQ508414, Bf-Vb-1 (Liu and Lo, 1994); Bungarus multicinctus PL 0702209A; Enhydrina schistosa P00610; Haemachatus haemachatus P00595; Laticauda colubria P10116; Laticauda laticaudata CAA68449; Laticauda semifasciata PLI BAB72247, PLIII P00612; Naja atra PL, CAA45372; PL-II, Q91133; Naja Kaouthia PLI, BAA36403, CMIII, P00597; Naja melanoleuca DE-I, P00599; Naja mossambica CM-I, P00602, CM-III, P00604; Naja oxiana, P25498; Naja pallida Nigexin, P14556; Naja sagittifera PLI, AAR16428, PLII, AAR00254; Naja sputatrix NPLA, Q92084; Notechis scutatus Notexin, P00608; Ophiophagus hannah PL-II, AF302907; Oxyuranus scutellatus Os-6 and 7, AAY47070-AAY47071; Pseudechis australis Pa11 P04056, Pa13 P04057; Pseudechis porphyriacus pseudexin A, P20258.

mamba). The functions of most of these short chain 3FTxs are not well defined.

KIns have usually been isolated from the venoms of elapids and true vipers but not from those of pitvipers (Zupunski et al., 2003). The molecular phylogenetic tree of the elapid venom KIns was constructed using a neurotoxin from mamba venom, Dtx-E, as an outgroup (Fig. 7). The tree topology revealed the clustering of elapid venom KIns under five groups, i.e. Australian Elapidae and sea snakes, kraits, Asian cobras, African cobras, and king cobras. This is apparently in accord with the phylogeographic relationships of these species. The data set used to build this tree does not include the B-chains of $\beta$-bungarotoxins, dendrotoxins, and viperid venom KIns. If included, they would be looped out from the majority of elapid KIn.

Interestingly, Wa KIn-II and III are linked to a chymotrypsin-inhibitor of king cobra venom and Wa KIn-I and IV are not associated with KIns of African cobras or other elapids. Thus, the cladograms of both venom PLAs and KIns (Figs. 5 and 7) suggest a close relationship between $W$. aegyptia and king cobra, and these molecular phylogeny trees might give new insights for the elapid biosystematics. In a recent species tree of elapids constructed based on sequences of mitochondrial DNA or other nuclear genes (Castoe et al., 2007), W. aegyptia was close or linked to $A$. scutatus rather than to king cobra, but the bootstrap value was too low to assure the relationship.

In conclusion, we solved the cDNA and the protein sequences of a total of seven $W$. aegyptia venom toxins (Figs. 3 and 4) and characterized the toxins. While its 3FTxs are either typical neurotoxins (e.g. Wa-III) or weak toxins (Wa-V), its PLAs and KIns are found to be similar or closely related to those of king cobra venom (Figs. 5 and 7). Morphological variations in the genus Walterinnesia across its distribution were recently examined, and the eastern populations (Turkey, Saudi Arabia, Iran) were found to differ consistently from those further west (Egypt, Israel, Jordan) in having lower scale row counts around the neck (21-23, vs. usually 25-27 in western populations), and in having a banded juvenile pattern (Nilson and Rastegar-Pouyani, 2007). Interestingly, king cobra has banded pattern for all juveniles and some of the adults, but lower scale row counts (15-19) than those of Walterinnesia. Taken the results together, king cobra (an Asian elapid) is likely to descent from African elapids in recent Natural history. Wa-PLAs are acidic enzymes and may inhibit platelets' function or affect the cardiovascular system (Al-Shammary et al., 1998), like king cobra venom PLAs (Huang et al., 1997a, b; Xu et al., 2003). Whether the 


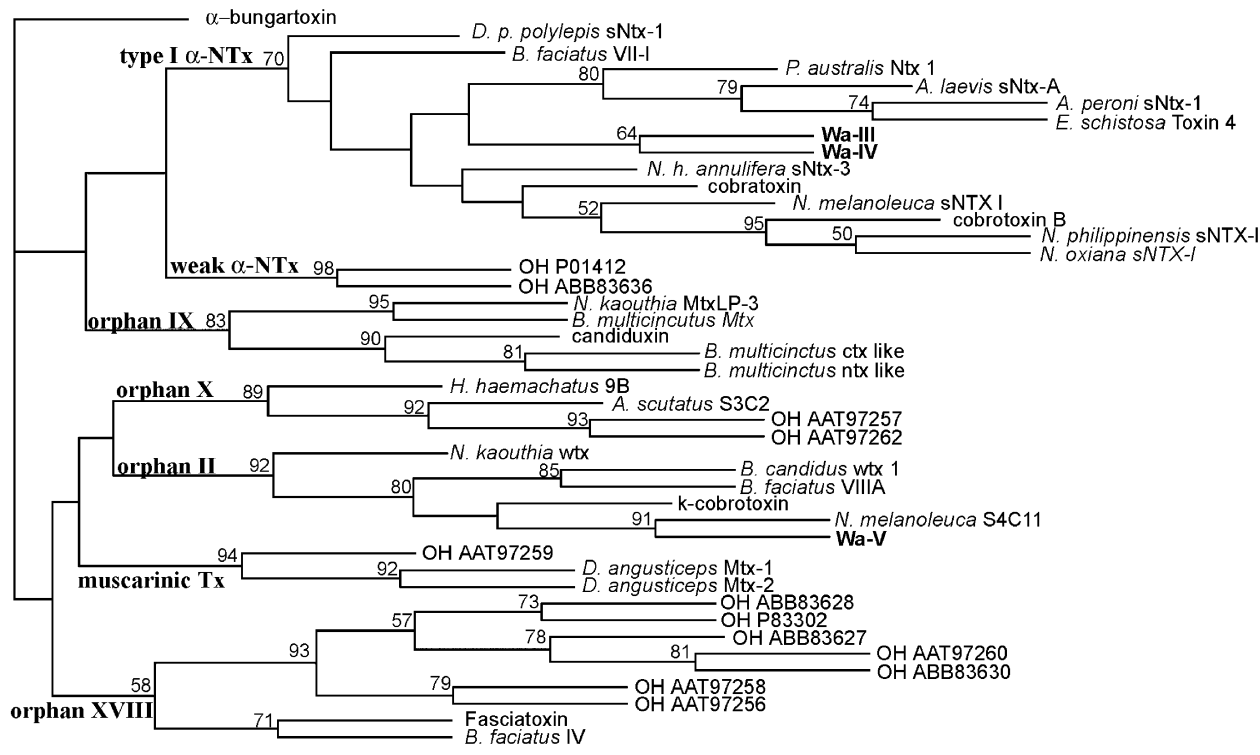

Fig. 6. Phylogenetic analysis of short chain 3FTxs. $\alpha$-Bungarotoxin is assigned as the outgroup. Bootstrap values, if $>50$, are shown at each node. Species, abbreviations, and accession numbers are: Acalyptophis peroni sNtx-1, AAV33393; Aipysurus laevis sNtx-A, P32879; Aspidelaps scutatus AsS3C2, P19003; B. candidus wtx 1, AAL30059, candiduxin 1, AAL30057; B. faciatus fasciatoxin, P14534, VII-1, P10808, VIIIa, A2CKF6, IV (Liu et al., 1991); B. multicinctus Ctxl, CAA71820, Bm X-2, Q9YGI0, Mtx, Q9W727; Dendroaspis angusticeps Mtx-1 and Mtx-2, P81030 and P18328; Dendroaspis p. polylepis sNtx-1, P01416; Enhydrina schistosa Toxin 4, P68415; H. haemachatus 9B P24778; N. atra $\kappa$-cobrotoxin CAA76846, cobrotoxin, P60770, cobrotoxin-b, P80958; N. haje annulifera Nha sNtx-3 P01420; N. kaouthia mtxlp-3, P82464, wtx P82935; N. melanoleuca SNTX-I P01424, S4C11 P01400; N. oxiana sNTX-I P01427; N. philippinensis sNTX-I P60773; Pseudechis australis Ntx-1 P25497.

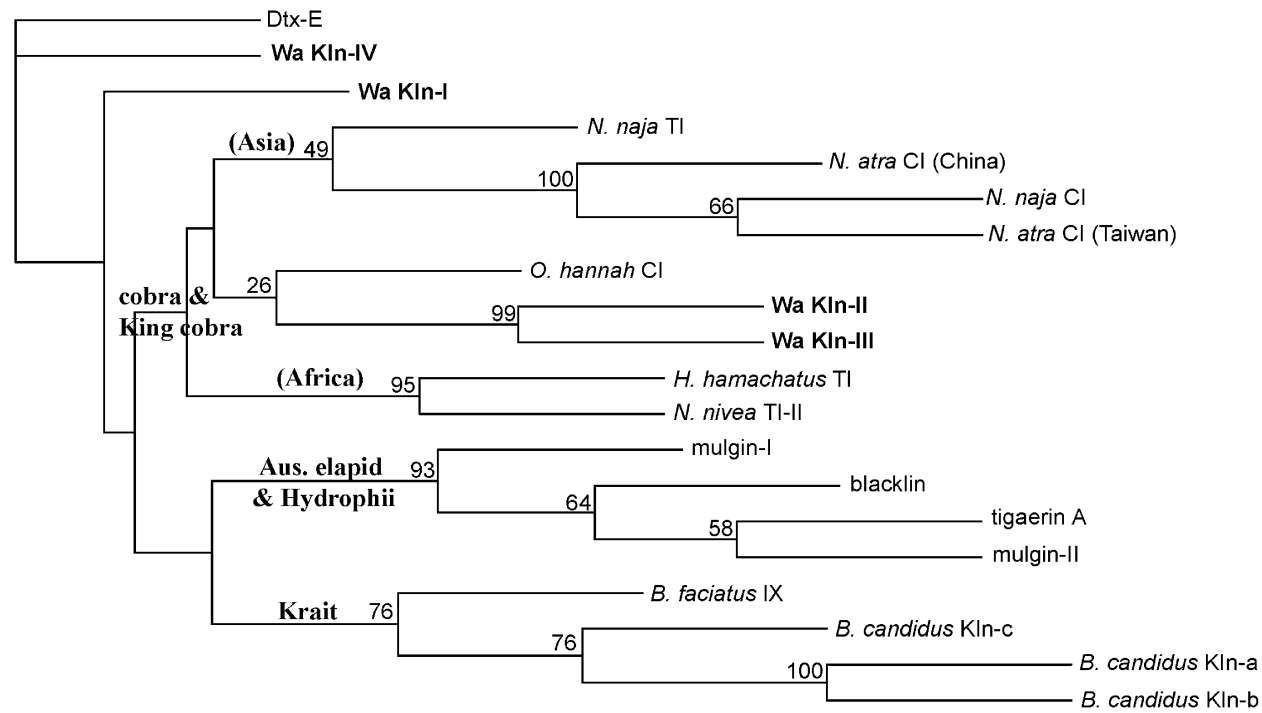

Fig. 7. Phylogenetic analysis of the venom KIns. D. p. polylepis Dtx-E (P00984) is assigned as the outgroup. Bootstrap values, if $>50$, are shown at each node. Species, abbreviations, and accession numbers are: B. candidus KIn-a-c, AAL30068-AAL30070; B. fasciatus IX P25660; H. haemachatus TI P00985; Chinese N. atra CI (Zhou et al., 2004), and Taiwanese N. atra CI, CAE51866; N. naja CI CAE51866, TI P20229; N. nivea TI-II P00986; N. scutatus, tigerin AAT45409; O. hannah CI P82966; P. australis mulgin I and II AAT45400-AAT45401; P. porphyriacus blacklin AAT45410.

W. aegyptia venom metalloprotease is similar to the P-III found in king cobra venom (Guo et al., 2007) remains to be investigated. Synergistic actions between these venom components possibly contribute to the rapid death reported for envenoming by either species. Further studies on the functions and properties of these venom components are underway.

\section{Acknowledgments}

Mass spectrometry analyses were performed at the National Core Facilities for Proteomics, Academia Sinica. This research was supported by grants from Academia Sinica and National Science Council, Taiwan, ROC. 


\section{References}

Al-Shammary, F.J., Al-Saleh, S.S., El-Shewemi, S.E., 1998. Ultrastructural variations of rat myocardium due to Walterinnesia aegyptia snake envenomation. J. Electron. Microsc. (Tokyo) 47, 637-643.

Altschul, S.F., Madden, T.L., Schäffer, A.A., Zhang, J., Zhang, Z., Miller, W., Lipman, D.J., 1997. Gapped BLAST and PSI-BLAST: a new generation of protein database search programs. Nucleic Acids Res. 25, 3389-3402.

Antil, S., Servent, D., Menez, A., 1999. Variability among the sites by which curaremimetic toxins bind to torpedo acetylcholine receptor, as revealed by identification of the functional residues of alphacobratoxin. J. Biol. Chem. 274, 34851-34858.

Antil-Delbeke, S., Gaillard, C., Tamiya, T., Corringer, P.J., Changeux, J.P., Servent, D., Ménez, A., 2000. Molecular determinants by which a long chain toxin from snake venom interacts with the neuronal alpha 7-nicotinic acetylcholine receptor. J. Biol. Chem. 275, 29594-29601.

Apostoluk, W., Otlewski, J., 1998. Variability of the canonical loop conformation in serine proteinases inhibitors and other proteins. Proteins: Struct. Funct. Genet. 32, 459-474.

Carlsson, F.H., 1975. Snake venom toxins. The primary structure of protein S4C11. A neurotoxin homologue from the venom of forest cobra (Naja melanoleuca). Biochim. Biophys. Acta 400, 310-321.

Castoe, T.A., Smith, E.N., Brown, R.M., Parkison, C.L., 2007. Higher-level phylogeny of Asian and American coralsnakes, their placement within the Elapidae (Squamata), and the systematic affinities of the enigmatic Asian coralsnake Hemibungarus calligaster (Wiegmann, 1834). Zool. J. Linn. Soc. 151, 809-831.

Chang, L.S., 2007. Genetic diversity in snake venom three-finger proteins and phospholipase $A_{2}$ enzymes. Toxin Rev. 26, 143-167.

Chang, L.S., Lin, S.K., Huang, H.B., Hsiao, M., 1999. Genetic organization of alpha-bungarotoxins from Bungarus multicinctus (Taiwan banded krait): evidence showing that the production of alpha-bungarotoxin isotoxins is not derived from edited mRNAs. Nucleic Acids Res. 27, 3970-3975.

Chang, L.S., Chung, C., Huang, H.B., Lin, S.K., 2001. Purification and characterization of a chymotrypsin inhibitor from the venom of Ophiophagus hannah (king cobra). Biochem. Biophys. Res. Commun. 283, 862-867.

Chen, Y.H., Wang, Y.M., Hseu, M.J., Tsai, I.H., 2004. Molecular evolution and structure-function relationships of crotoxin-like and asparagine 6 -containing phospholipases $A_{2}$ in pit viper venoms. Biochem. J. 381, 25-34.

Cheng, Y.C., Yan, F.J., Chang, L.S., 2005. Taiwan cobra chymotrypsin inhibitor: cloning, functional expression and gene organization. Biochim. Biophys. Acta 1747, 213-220.

Danse, J.M., Gasparini, S., Menez, A., 1997. Molecular biology of snake venom phospholipases $A_{2}$. In: Kini, R.M. (Ed.), Venom Phospholipase $\mathrm{A}_{2}$ Enzyme: Structure, Function and Mechanism. Wiley, UK, pp. 29-71.

Davidson, F.F., Dennis, E.A., 1990. Evolutionary relationships and implications for the regulation of phospholipase $A_{2}$ from snake venom to human secreted forms. J. Mol. Evol. 31, 228-238.

Felsenstein, J., 1985. Confidence limits on phylogenies: an approach using the bootstrap. Evolution 39, 783-791.

Felsenstein, J., 1992. Phylip: The Phylogeny Interfence Package, Version 3.573. Computer program distributed by the University of Washington, Department of Genetics, Seattle, USA.

Filippovich, I., Sorokina, N., Masci, P.P., Jersey, J.D., Whitaker, A.N., Winzor, D.J., Gaffney, P.J., Lavin, M.F., 2002. A family of textilinin genes, two of which encode proteins with antihaemorrhagic properties. Br. J. Haematol. 119, 376-384.

Flight, S., Johnson, L., Trabi, M., Gaffney, P., Lavin, M., de Jersey, J., Masci, P.P., 2005. Comparison of textilinin-1 with aprotinin as serine protease inhibitors and as antifibrinolytic agents. Pathophysiol. Haemost. Thromb. 34, 188-193.

Fry, B.G., Wuster, W., Kini, R.M., Brusic, V., Khan, A., Venkataraman, D., Rooney, A.P., 2003. Molecular evolution and phylogeny of elapid snake venom three-finger toxins. J. Mol. Evol. 57, 110-129.

Gitter, S., de Vries, A., 1968. Symptomatology, pathology and treatment of bites of near eastern, European and north African snakes. In: Burchel, W., Buckley, E., Delofeu, V. (Eds.), Venomous Animals and their Venoms, vol. 1. Academic Press, New York, pp. 359-401.

Grzesiak, A., Helland, R., Smalas, A.O., Krowarsch, D., Dadlez, M., Otlewski, J., 2000. Substitutions at the P1' position in BPTI strongly affect the association energy with serine proteinases. J. Mol. Biol. 301, 205-217.

Guo, X.X., Zeng, L., Lee, W.H., Zang, Y., Jin, Y., 2007. Isolation and cloning of a metalloprotease from king cobra snake venom. Toxicon 49 , 49954-49965.
Huang, M.Z., Gopalakrishnakone, P., Chung, M.C., Kini, R.M., 1997a. Complete amino acid sequence of an acidic, cardiotoxic phospholipase $\mathrm{A}_{2}$ from the venom of Ophiophagus hannah (king cobra): a novel cobra venom enzyme with "pancreatic loop". Arch. Biochem. Biophys. 15, 338, 150-156.

Huang, M.Z., Gopalakrishnakone, P., Kini, R.M., 1997b. Role of enzymatic activity in the antiplatelet effects of a phospholipase $A_{2}$ from Ophiophagus hannah snake venom. Life Sci. 61, 2211-2217.

Ismail, M. Abd-Elsalam, M.A. Al-Ahaidib, M.S., 1998. Pharmacokinetics of ${ }^{125}$ I-labelled Walterinnesia aegyptia venom and its specific antivenins: flash absorption and distribution of the venom and its toxin versus slow absorption and distribution of $\operatorname{IgG}, \mathrm{F}\left(\mathrm{ab}^{\prime}\right) 2$ and $\mathrm{F}(\mathrm{ab})$ of the antivenin. Toxicon 36, 93-114.

Karlsson, E., 1979. Chemistry of protein toxins in snake venoms. In: Lee, C.Y. (Ed.), Snake Venoms, Handbook of Experimental Pharmacology, vol. 52. Springer, Berlin, pp. 159-212.

Kini, R.M., 2002. Molecular moulds with multiple missions: functional sites in three-finger toxins. Clin. Exp. Pharmacol. Physiol. 29, 815-822.

Laskowski Jr., M., Kato, I., 1980. Protein inhibitors of proteinases. Annu. Rev. Biochem. 49, 593-626.

Lee, C.Y., Tsai, M.C., 1972. Does the desert black snake inhibit release of acetylcholine from motor nerve endings. Toxicon 10, 659-660.

Lee, C.Y., Chen, Y.M., Mebs, D., 1976. Chromatographic separation of the venom of Egyptian black snake (Walterinnesia aegyptia) and pharmacological characterization of its components. Toxicon 14, 275-281.

Li, J., Zhang, H., Liu, J., Xu, K., 2006. Novel genes encoding six kinds of three-finger toxins in Ophiophagus hannah (king cobra) and function characterization of two recombinant long-chain neurotoxins. Biochem. J. 398, 233-242.

Liu, C.S., Lo, T.B., 1994. Chemical studies of Bungarus fasciatus venom. J. Chin. Biochem. 23, 69-75.

Liu, C.S., Chen, J.P., Chang, C.M., Chen, S.W., Lo, T.B., 1991. Amino acid sequence of a fasciatoxin-homologue, fasciatoxin-II in the venom of Bungarus fasciatus (banded karit). J. Chin. Biochem. Soc. 20, 33-39.

Liu, X., Zhu, H., Huang, B., Rogers, J., Yu, B.Z., Kumar, A., Jain, M.K. Sundaralingam, M., Tsai, M.D., 1995. Phospholipase $A_{2}$ engineering. Probing the structural and functional roles of $\mathrm{N}$-terminal residues with site-directed mutagenesis, X-ray, and NMR. Biochemistry 34, 7322-7334.

Maniatis, T., Fritsch, E.F., Sambrook, J., 1989. Molecular Cloning, a Laboratory Manual. Cold Spring Harbor Laboratory, Cold Spring Harbor, New York.

Menez, A., 1998. Functional architectures of animal toxins: a clue to drug design? Toxicon 36, 1557-1572.

Millers, E.K., Masci, P.P., Lavin, M.F., de Jersey, J., Guddat, L.W., 2006. Crystallization and preliminary X-ray analysis of a Kunitz-type inhibitor, textilinin-1 from Pseudonaja textilis textilis. Acta Crystallogr. Sec. F 62, 642-645.

Mullis, K.B., Faloona, F.A., 1987. Specific synthesis of DNA in vitro via a polymerase-catalyzed chain reaction. Methods Enzymol. 155, 335-350.

Nilson, G., Rastegar-Pouyani, N., 2007. Walterinnesia aegyptia Lataste, 1887 (Ophidia: Elapidae) and the status of Naja morgani Mocquard 1905. Russ. J. Herpet. 14, 7-14.

Ohno, M., Menez, R., Ogawa, T., Danse, J.M., Shimohigashi, Y., Fromen, C., Ducancel, F., Zinn-Justin, S., Le Du, M.H., Boulain, J.C., Tamiya, T., Menez, A., 1998. Molecular evolution of snake toxins: is the functional diversity of snake toxins associated with a mechanism of accelerated evolution. Prog. Nucleic Acids Res. Mol. Biol. 59, 307-364.

Pillet, L., Tremeau, O., Ducancel, F., Drevet, P., Zinn-Justin, S., Pinkasfeld, S., Boulain, J.C., Menez, A., 1993. Genetic engineering of snake toxins. Role of invariant residues in the structural and functional properties of a curaremimetic toxin, as probed by site-directed mutagenesis. J. Biol. Chem. 268, 909-916.

Rajagopalan, N., Pung, Y.F., Zhu, Y.Z., Wong, P.T., Kumar, P.P., Kini, R.M., 2007. $\beta$-Cardiotoxin: a new three-finger toxin from Ophiophagus hannah (king cobra) venom with beta-blocker activity. FASEB J. 21, 3685-3695.

Samejima, Y., Aoki-Tomomatsu, Y., Yanagisawa, M., Mebs, D., 1997. Amino acid sequences of two neurotoxins from the venom of the Egyptian black snake (Walterinnesia aegyptia). Toxicon 35, 151-157.

Schechter, I., Berger, A., 1967. On the size of the active site in proteases. I. Papain. Biochem. Biophys. Res. Commun., 157-162.

Simon, T., Bdolah, A., 1980. Isolation of phospholipase $A_{2}$ from the venom of the desert black snake Walterinnesia aegyptia. Toxicon 18, 369-373.

Slowinski, J.B., Knight, A., Rooney, A.P., 1997. Inferring species trees from gene trees: a phylogenetic analysis of the Elapidae (Serpentes) based 
on the amino acid sequences of venom proteins. Mol. Phylogenet. Evol. 8, 349-362.

Thompson, J.D., Higgins, D.G., Gibson, T.J., 1994. Improving the sensitivity of progressive multiple sequence alignment through sequence weighting, position-specific gap penalties and weight matrix choice. Nucleic Acids Res. 22, 4673-4680.

Tremeau, O., Lemaire, C., Drevet, P., Pinkasfeld, S., Ducancel, F., Boulain, J.C., Ménez, A., et al., 1995. Genetic engineering of snake toxins. The functional site of erabutoxin a, as delineated by site-directed mutagenesis, includes variant residues. J. Biol. Chem. 270, 9362-9370.

Tsai, I.H., Hsu, H.Y., Wang, Y.M., 2002. A novel phospholipase $A_{2}$ from the venom gland of Bungarus candidus: cloning and sequence-comparison. Toxicon 40, 1363-1367.

Tsai, I.H., Chen, Y.H., Wang, Y.M., 2004. Comparative proteomics and subtyping of venom phospholipases $\mathrm{A}_{2}$ and disintegrins of Protobothrops pit vipers. BBA-Prot. Proteom. 1702, 111-119.
Tsetlin, V.I., Hucho, F., 2004. Snake and snail toxins acting on nicotinic acetylcholine receptors: fundamental aspects and medical applications. FEBS Lett. 557, 9-13.

White, S.P., Scott, D.L., Otwinowski, Z., Gelb, M.H., Sigler, P.B., 1990 Crystal structure of cobra-venom phospholipase $\mathrm{A}_{2}$ in a complex with a transition-state analogue. Science 250, 1560-1563.

Xu, S., Gu, L., Wang, Q., Shu, Y., Song, S., Lin, Z., 2003. Structure of king cobra phospholipase $A_{2}$ determined from hemihedrally twinned crystal. Acta Crystallogr. D 59, 1574-1581.

Zhou, X.D., Jin, Y., Lu, Q.M., Li, D.S., Zhu, S.W., Wang, W.Y., Xiong, Y.L., 2004. Purification, characterization and primary structure of a chymotrypsin inhibitor from Naja atra venom. Comp. Biochem. Physiol. 137B, 219-224.

Zupunski, V., Kordis, D., Gubensek, F., 2003. Adaptive evolution in the snake venom Kunitz/BPTI protein family. FEBS Lett. 547, 131-136. 